

\title{
DETERMINACIÓN DEL COSTO DE CONSTRUCCIÓN DE LAS DIFERENTES CLASIFICACIONES PARA UNA VIVIENDA SUSTENTABLE EN LA CIUDAD DE CUENCA, ECUADOR
}

\author{
DETERMINATION OF THE CONSTRUCTION COST OF THE DIFFERENT \\ CLASSIFICATIONS FOR A SUSTAINABLE DWELLING IN THE CITY OF \\ CUENCA, ECUADOR
}

\author{
JORGE RENE VASQUEZ PALACIOS \\ Universidad de Cuenca \\ Cuenca Ecuador \\ jorgevasquezp1990@hotmail.com
}

\author{
JUAN FELIPE QUESADA MOLINA \\ Universidad de Cuenca \\ Cuenca Ecuador \\ felipe.quesada@ucuenca.edu.ec
}

\section{RESUMEN}

En Ecuador existe un elevado porcentaje de viviendas con problemas ambientales, debido a la falta de una práctica habitual de incorporar criterios de confort y eficiencia energética, que patentiza la necesidad de implementar estrategias sustentables en los procesos de construcción. El estudio aquí expuesto analiza una vivienda unifamiliar en la ciudad de Cuenca (Ecuador) y determina los costos referenciales de construcción (por metro cuadrado) de los diferentes niveles sustentables que pudiera alcanzar la vivienda con respecto a su semejante convencional. El trabajo se desarrolla en cuatro etapas: (1) desarrollo de una metodología apropiada para identificar los costos; (2) estudio cualitativo sobre la percepción de la vivienda sustentable; (3) estudio cuantitativo de los costos de construcción y el valor agregado ecuatoriano (VAE). A las distintas opciones de viviendas sustentables se las clasifica en una escala de $\mathrm{A}$ a $\mathrm{C}$, dependiendo de la cantidad de estrategias que se incorporen, mientras que a la vivienda convencional se le asigna la clasificación D. Se concluye que las viviendas sustentables presentan un incremento de costo, con respecto a la convencional, del $33.14 \%(\mathrm{~A}), 15.56 \%$ (B) y $4.32 \%$ (C), con una reducción del VAE del 15.75\%, 4.01\% y 3.74\%, respectivamente

\section{Palabras clave}

viabilidad económica, viviendas unifamiliares, costos de prácticas sustentables, costo inicial, CuencaEcuador

\section{ABSTRACT}

In Ecuador, a high percentage of homes have environmental failures due to the lack of customary incorporation of comfort and energy efficiency criteria, which demonstrates the need to implement sustainable strategies in construction processes. This study analyzes a single-family dwelling in the city of Cuenca (Ecuador), and determines the referential construction costs (per square meter) of the different sustainability levels that the home could attain in comparison with a conventional counterpart. The research was carried out in four stages: (1) the development of an appropriate methodology to identify costs; (2) a qualitative study on the perception of sustainable housing; and (3) a quantitative study of construction costs and Ecuadorian Added Value (VAE). The different sustainable

housing options were classified on a scale from $A$ to $C$ depending on the number of strategies incorporated, while conventional housing was classified as D. It was concluded that compared to conventional housing, sustainable dwellings present a cost increase of $33.14 \%(A), 15.56 \%(B)$, and $4.32 \%$ (C), and a VAE reduction of $15.75 \%, 4.01 \%$ and $3.74 \%$, respectively 


\section{INTRODUCCIÓN}

El Programa de las Naciones Unidas para el Medio Ambiente, UNEP SBCI (2009), manifiesta que el sector de la construcción es uno de los principales contribuyentes de emisiones de gases de efecto invernadero. La región de América Latina es susceptible a los efectos derivados del cambio climático y su nivel de aportación de emisiones se incrementa (Uribe, 2015).

Los edificios, en general, tienen un impacto significativo en el medio ambiente (Oneill y Gibbs, 2014): un análisis comparativo mostró que los diseños de edificaciones sustentables exhibieron un ahorro del $16.3 \%$ en su ciclo de vida y hasta un $50 \%$ de ahorro anual de energía (Assad et al., 2015), y una reducción del $16 \%$ en promedio de su huella de carbono (Kneifel, 2010).

En este sentido, los edificios sustentables son una mejora significativa sobre los edificios convencionales del pasado, ya que consumen menos energía, materiales y agua, y a su vez proporcionan mejores entornos de trabajo y vida saludable.

La sustentabilidad busca el mayor beneficio dados los costos de las prácticas ecológicas, lo cual implica un equilibrio entre mejorar, tanto el desempeño ambiental operativo de la vivienda como la calidad de vida social de los residentes, y reducir el costo del ciclo de vida (García Erviti, Armengot Paradinas y Ramírez Pacheco, 2015; Karatas y El-Rayes, 2015; Zore, Čuček y Kravanja, 2017) (Figura 1).

En la construcción, los costos ecológicos hacen referencia a los generados por la implementación de estrategias sustentables, lo cual optimiza el desempeño ambiental, pero generalmente un aumento del costo inicial (Karatas y El-Rayes, 2015)

Existen numerosos estudios que se enfocan en el desempeño ambiental de las viviendas; sin embargo, en lo económico no se presta mayor atención y existe una incertidumbre sobre las estimaciones de costos de construcción, y una necesidad por mejorar los modelos de predicción de costos de edificaciones sostenibles (Alshamrani, 2017; Berardi, 2013), ya que para que un edificio sea considerado sostenible debe promover una perspectiva a largo plazo de su valor económico, pues constituye uno sus 10 principios (Conseil International du Bâtiment, 2010). Esta perspectiva económica considera el ciclo de vida de una edificación, que involucra la construcción, la operación, el mantenimiento y el final del ciclo (Berardi, 2013).

Al comparar los costos de construcción de edificios convencionales y sustentables, se argumenta que el alto costo de los edificios sustentables es la principal razón que desmotiva a los propietarios a asumir proyectos en este campo (Hwang y Tan, 2012). Contrariamente a este

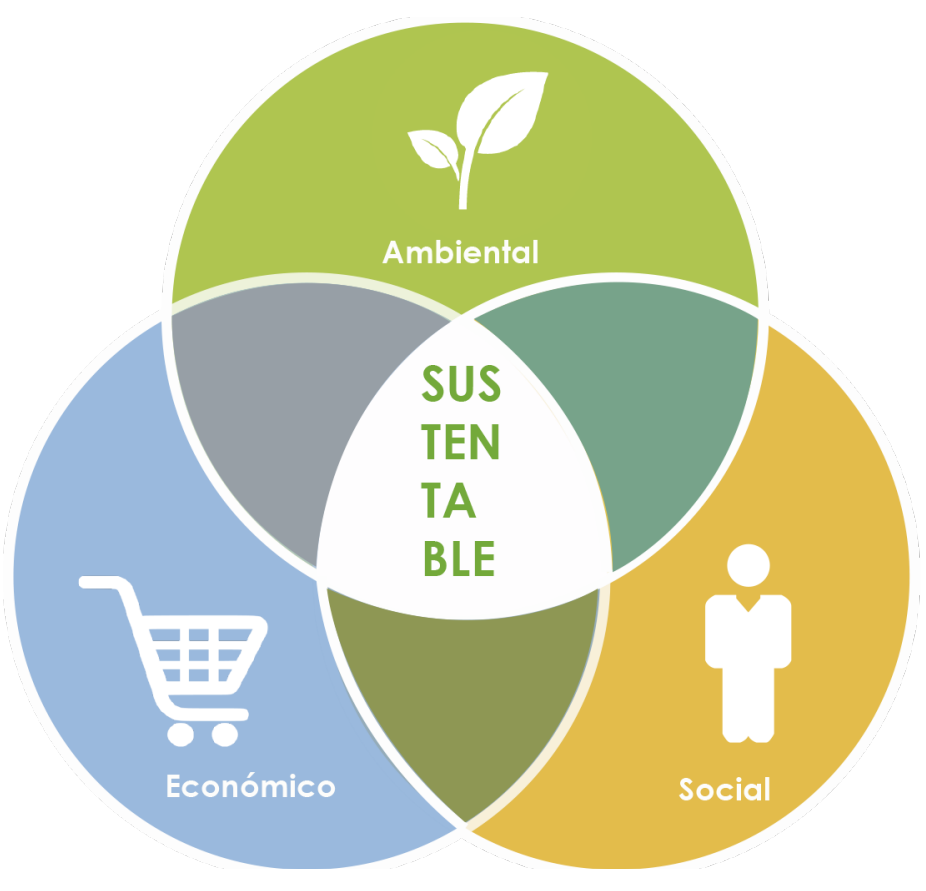

Figura 1. Diagrama de Venn del modelo de desarrollo sustentable. Fuente: Elaboración de autores.

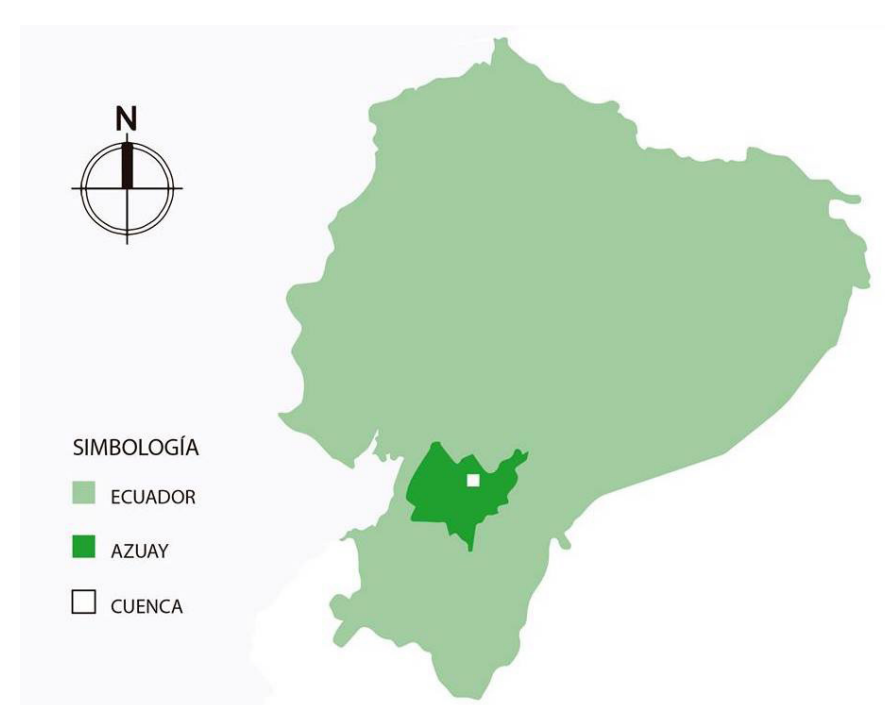

Figura 2. Ubicación geográfica de la ciudad de Cuenca, Azuay, Ecuador. Fuente: Elaboración de autores.

pensamiento, se menciona que la percepción de los altos costos de construcción sustentable están sobreestimados y, a la vez, el ahorro económico y el beneficio al medio ambiente a largo plazo están siendo minimizados (Bartlett y Howard, 2000). La construcción sustentable implica considerar los costos sociales, ambientales y económicos dentro de todo el ciclo de vida de la edificación, si se amplía la percepción del costo sustentable, en realidad es más barato optar por lo sustentable. 


\begin{tabular}{|c|c|c|c|c|}
\hline ESTUDIO & AUTOR & METODOLOGÍA & $\begin{array}{c}\text { ENCUESTA / } \\
\text { ENTREVISTA }\end{array}$ & $\begin{array}{c}\text { COMPARACIÓN } \\
\text { DE PRESUPUESTOS }\end{array}$ \\
\hline $\begin{array}{c}\text { The costs and financial benefits } \\
\text { of green buildings: A report to } \\
\text { California's Sustainable Building Task } \\
\text { Force. }\end{array}$ & (Kats et al., 2003) & Cualitativa & $\cdot$ & \\
\hline $\begin{array}{c}\text { Value case for sustainable building in } \\
\text { New Zealand }\end{array}$ & $\begin{array}{c}\text { (Fullbrook, Jackson y } \\
\text { Finlay, 2006) }\end{array}$ & Cuantitativa & & \\
\hline The cost of green in NYC. & $\begin{array}{c}\text { (Urban Green Council, } \\
\text { 2009) }\end{array}$ & Cuantitativa & & \\
\hline $\begin{array}{c}\text { Greening our built environment: } \\
\text { Costs, benefits, and strategies. }\end{array}$ & $\begin{array}{c}\text { (Kats, Braman y James, } \\
\text { 2010) }\end{array}$ & Cualitativa & & \\
\hline $\begin{array}{c}\text { Construction costs comparison } \\
\text { between 'green' and conventional } \\
\text { office buildings. }\end{array}$ & (Rehm y Ade, 2013) & $\begin{array}{c}\text { Cualitativa - } \\
\text { Cuantitativa }\end{array}$ & - & \\
\hline
\end{tabular}

Tabla 1. Metodologías desarrolladas en investigaciones de costos de construcción de edificaciones sustentables.

Fuente: Elaboración de autores.

Por otra parte, en el Ecuador no existe una práctica habitual de incorporar criterios de confort y eficiencia energética en la construcción (Instituto Nacional de Eficiencia Energética y Energías Renovables, 2014), lo cual refleja un elevado porcentaje de viviendas con problemas ambientales, sin embargo la Constitución vigente reconoce el derecho a "un hábitat seguro y saludable, a una vivienda adecuada y digna, un ambiente sano y ecológicamente equilibrado" (Asamblea Constituyente del Ecuador, 2008).

El presente estudio constituye un aporte, ya que determina si existen costos adicionales por la implementación de estrategias sustentables en una vivienda unifamiliar de la ciudad, permitiendo hacer una predicción de los costos iniciales de una vivienda sustentable y compararla con su semejante convencional.

La investigación se desarrolló en la ciudad de Cuenca, provincia del Azuay, situada en la región de la Sierra Ecuatoriana (Figura 2), la que cuenta con una temperatura promedio de $13,1^{\circ} \mathrm{C}$, índice de calor de $12,9^{\circ} \mathrm{C}$, humedad de $79 \%$ y lluvia anual de 610,2mm (EXA, 2017).

Se analiza una vivienda de tipo unifamiliar, pues representa la tipología predominante en la ciudad, al constituir el $76 \%$ del total (Instituto Nacional de Estadísticas y Censos, 2010a).

Se adoptó como estrategia de investigación una metodología mixta (encuesta y medición de variables en viviendas), la cual enlaza diversas perspectivas cualitativas y cuantitativas del fenómeno.
Además, como los edificios sustentables son concebidos de forma que empleen materiales locales (Umar, Khamidi y Tukur, 2012) y aprovechen los recursos ambientales mediante la adaptación a su entorno (Gunawansa y Kua, 2014), se plantea evaluar el valor agregado ecuatoriano (VAE), que constituye una política del Ecuador que da preferencia a la producción nacional y permite calcular en cada producto el porcentaje de componente ecuatoriano que incorpora (Servicio Nacional de Contratación Pública, 2015b).

\section{MATERIALES Y MÉTODOS}

\section{DESARROLLO DE LA METODOLOGÍA}

Con base en una revisión bibliográfica donde se analizan y determinan los costos de construcción de edificaciones sustentables, se identifica y compara las metodologías empleadas (Tabla 1); de esta manera, se selecciona la metodología de Rehm y Ade (2013) pues brinda mayor profundidad de estudio al enlazar la perspectiva cualitativa y cuantitativa.

\section{ANÁLISIS CUALITATIVO}

Se desarrolla a través de una encuesta estructurada aplicada a cabezas de hogares en el sector residencial del cantón Cuenca, con el objetivo de estimar la percepción de la sociedad en cuanto al interés y a costos asociados a la construcción de viviendas sustentables.

El diseño específico del muestreo estadístico aplicado es de tipo probabilístico, polimetálico y aleatorio, y la selección se realiza en base al número de viviendas urbanas existentes en el cantón. 
El tamaño de la muestra determinada es de 280 viviendas de un total de 273.186 conforme el Censo 2010 de Población y Vivienda en el Ecuador (INEC, 2010b), por lo cual se considera un nivel de confianza en los resultados de 95\% y un error absoluto de 0,06. Esta muestra se encuentra distribuida y clasificada por niveles socioeconómicos en las 15 parroquias urbanas del cantón Cuenca.

\section{ANÁLISIS CUANTITATIVO}

El análisis cuantitativo conforma la segunda etapa de la investigación, y consiste en el análisis y comparación del presupuesto de una edificación sustentable frente a una convencional. En esta etapa se optó por un diseño de investigación no experimental, aplicando un estudio transaccional.

\section{Primera etapa: Desarrollo de Metodología.}

La metodología desarrollada por Rehm y Ade (2013) recopila los datos de costos reales de edificaciones sustentables en construcción 0 de proyectos concluidos en un periodo cercano al estudio. Por otro lado, los costos de construcción de las edificaciones convencionales provienen de las principales fuentes referenciales en el país. Determinado el origen de estos, se elaboran los presupuestos para las diferentes tipologías de edificaciones $y$, además en esta etapa, para que los presupuestos tengan características similares y sean comparables, se retiran de los proyectos los costos de obras interiores exclusivas de cada edificio, obras previas, costo del terreno y costos derivados de préstamos bancarios.

Los presupuestos ajustados son analizados estadísticamente con la prueba no paramétrica de Wilcoxon (1945) de pares emparejados, con el fin de determinar la variación del costo de construcción de una edificación sustentable respecto de su semejante convencional.

Para este análisis se seleccionó un caso de estudio que reuniera las características de una vivienda convencional y se le implementó criterios y prácticas constructivas de sustentabilidad. La vivienda fue modelada con los parámetros de diseño sustentables establecidos en el proyecto de investigación "Método de Certificación de la Construcción Sustentable de la Vivienda" (Vivienda sustentable y segura Cuenca, 2017).

Los costos de construcción de una vivienda convencional y sustentable se obtienen de una misma fuente referencial de costos de construcción local como el boletín técnico de (Cámara de la Construcción de Cuenca, 2016).

Los presupuestos están conformados por capítulos que representan conjuntos de actividades de construcción relacionadas entre sí por período de ejecución, técnica constructiva o materiales empleados. Esta configuración servirá para comparar y determinar los indicadores de variación entre los diferentes presupuestos.

El método de comparación no paramétrico de pares de Wilcoxon (1945) es aplicado a muestras que deben tener más de 6 pares de observaciones; requerimiento mínimo del cual no dispone este estudio, por lo que se opta por realizar una comparación simple de pares dada la cantidad de observaciones disponibles. La comparación adoptada consiste en confrontar los costos por capítulos y el costo total por metro cuadrado de la vivienda referencial sustentable con los valores semejantes de la vivienda unifamiliar convencional seleccionada como caso de estudio.

\section{Segunda etapa: Aplicación.}

Se seleccionó el Boletín Técnico de la Cámara de Construccion de Cuenca (2016), edición del mes de noviembre, como fuente referencial de costos para el análisis de la edificación convencional y sustentable, debido a que su metodología de cálculo refleja: las condiciones de construcción local, los índices de precios de materiales nacionales y locales, la tabla salarial de mano de obra, el rendimiento de la mano de obra local y la disposición del mercado para estimar el costo actual de cada rubro.

Con el fin de completar los presupuestos de la vivienda de estudio se decidió estimar los costos no presentes en dicha publicación, elaborándolos con las mismas consideraciones de cálculo que los costos del boletín seleccionado.

Para la modelación de los costos y presupuestos, en este estudio se utilizó el software especializado de ingeniería de Costos InterPro 2010 versión 2.4.1 Módulo de Ofertas, programa que determina el costo de una actividad de construcción concreta por unidad de cálculo.

Las cantidades de obra de las diferentes actividades de construcción que conforman los presupuestos analizados, así como el cálculo del área total de construcción, provienen de la modelación digital y del levamiento llevado a cabo para el caso de estudio.

Al considerar que los edificios sustentables se conciben priorizando el aprovechamiento de materiales propios de su localidad (Umar, Khamidi y Tukur, 2012), esta investigación calcula el indicador definido como $\mathrm{VAE}$, en aras de determinar el grado de componente ecuatoriano que posee un producto.

Los criterios, metodología y cuadros de cálculo empleados para determinar el VAE se encuentran detallados en las resoluciones No. RE-SERCOP-2015-031 
(Secretaría Nacional de Contratación Pública, 2015a) y No. RE-SERCOP-2015-033 (SERCOP, 2015b).

\section{Tercera etapa: Selección de caso de estudio.}

Se selecciona, en esta fase, una vivienda unifamiliar convencional que forma parte de un grupo mayor de 11 analizadas dentro del proyecto de investigación "Método de Certificación de la Construcción Sustentable de la Vivienda", del cual forma parte el presente estudio.

La vivienda $n^{\circ} 6$ (Tabla 2), que se ubica en la parroquia urbana Machángara, del cantón Cuenca, responde a la tipología analizada y, al ser un modelo continuo de análisis y desarrollo dentro del proyecto de investigación, se convierte en un referencial de estudio.

\section{Cuarta etapa: clasificación de viviendas sustentables.} Con el propósito de establecer el desempeño sustentable, se optó por los niveles de referencia de las viviendas sustentables establecidos por Quesada (2014):

- Práctica Estándar (C)

- Práctica Mejor (B)

- Práctica Superior (A)

Para cada nivel de desempeño, el proyecto de investigación "Método de Certificación de la construcción sustentable de la vivienda" (Vivienda sustentable y segura Cuenca, 2017) define un grupo de estrategias sustentables que las viviendas en la ciudad de Cuenca deben cumplir.

\section{Quinta etapa: Estrategias aplicadas.}

Las estrategias sustentables incorporadas en las viviendas eficientes referenciales se determinaron en los estudios: "Eficiencia Energética en el Sector Residencial de la Ciudad de Cuenca, Ecuador" (Baquero y Quesada, 2016), "Evaluación del consumo energético en la fase de uso de la edificación residencial en Cuenca, diseño de estrategias para un modelo de vivienda eficiente" (Calle y Ortiz, 2016) y "Sustentabilidad de agua en viviendas de la ciudad de Cuenca" (Molina y Orellana, 2017), los cuales se desarrollan en los ámbitos de energía, envolvente y agua, respectivamente.

En la Tabla 3, se resumen las estrategias consideradas que, en ciertos casos, presentan varias opciones. En función de esto, se presupuesta todas las alternativas planteadas y se obtiene como valores referenciales rangos de Costos y VAE. Un ejemplo concreto es la cubierta de la vivienda, la cual presenta 8 alternativas distintas de aislamiento generando un rango de costos entre $\$ 28,67$ a $\$ 72,38$ USD (dólares americanos) y un VAE del $63,53 \%$ al $95,75 \%$.

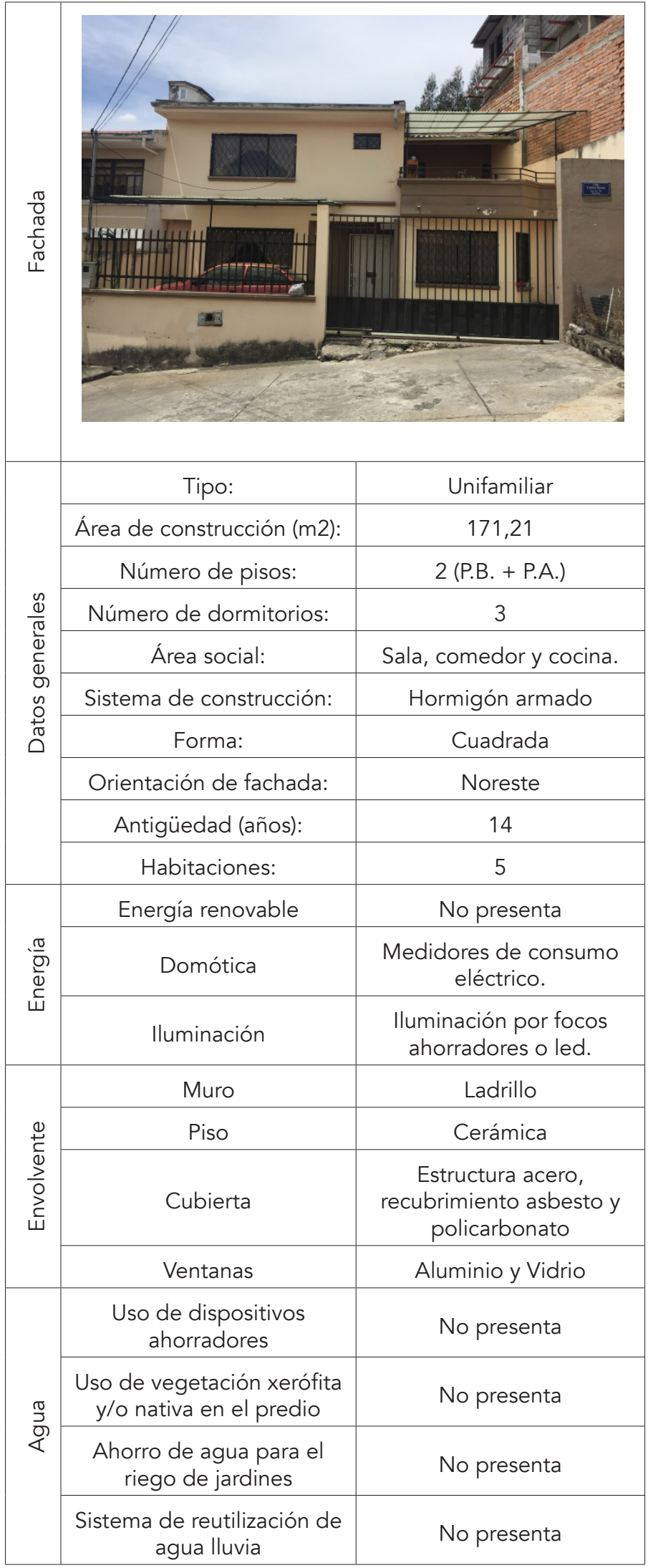

Tabla 2. Vivienda $n^{\circ} 6$ (caso de estudio). Fuente: Elaboración de los autores. 


\begin{tabular}{|c|c|c|c|c|c|}
\hline \multicolumn{2}{|c|}{ Ámbito de aplicación } & Estrategia & $\begin{array}{l}\text { Categorías de } \\
\text { aplicación }\end{array}$ & $\begin{array}{l}\text { Costo } \\
\text { (USD) }\end{array}$ & $\begin{array}{l}\text { Valor agregado } \\
\text { ecuatoriano } \\
\text { (\%) }\end{array}$ \\
\hline \multirow{10}{*}{ Energía } & \multirow{2}{*}{$\begin{array}{l}\text { Energía } \\
\text { renovable }\end{array}$} & $\begin{array}{c}\text { Paneles solares fotovoltaicos para } \\
\text { generar energía eléctrica. }\end{array}$ & A & 1849,69 & $10,50 \%$ \\
\hline & & $\begin{array}{l}\text { Paneles solares para el } \\
\text { calentamiento de agua. }\end{array}$ & A & 1040,24 a 2697,37 & $08,85 \%$ a $48,76 \%$ \\
\hline & \multirow{7}{*}{ Domótica } & Sistema de control domótica. & A & 1215,77 & $0,34 \%$ \\
\hline & & Sensores multifunción domótica. & A & 92,51 & $2,21 \%$ \\
\hline & & $\begin{array}{c}\text { Sistema eficiente de iluminación } \\
\text { domótica. }\end{array}$ & A & 212,95 & $18,36 \%$ \\
\hline & & $\begin{array}{l}\text { Controlador automático de } \\
\text { persianas y cortinas. }\end{array}$ & A & 128,00 & $1,60 \%$ \\
\hline & & $\begin{array}{c}\text { Sistema de detección, gestión } \\
\text { y control del consumo de los } \\
\text { electrodomésticos. }\end{array}$ & A & 143,50 & $0,71 \%$ \\
\hline & & Medidores de consumo eléctrico. & A & 191,87 & $1,33 \%$ \\
\hline & & $\begin{array}{c}\text { Sensores de corriente de } \\
\text { consumo eléctrico }\end{array}$ & A & 34,34 & $2,98 \%$ \\
\hline & Iluminación & $\begin{array}{c}\text { Iluminación por focos ahorradores } \\
\text { o led. }\end{array}$ & $B-C$ & 51,22 a 76,86 & $48,99 \%$ a $73,52 \%$ \\
\hline \multirow{4}{*}{ Envolvente } & Muro & Aislamiento térmico de muro. & $A-B$ & 38,85 a74,03 & $65,47 \%$ a $90,68 \%$ \\
\hline & Piso & Aislamiento térmico de piso. & $A-B$ & 45,83 a 55,74 & $89,12 \%$ a $93,16 \%$ \\
\hline & Cubierta & Aislamiento térmico de cubierta. & $A-B$ & 28,67 a 72,38 & $59,44 \%$ a $92,55 \%$ \\
\hline & Ventanas & Aislamiento térmico de ventana. & $A-B$ & 80,53 a 125,14 & $57,14 \%$ a $89,52 \%$ \\
\hline \multirow{9}{*}{ Agua } & \multirow{4}{*}{$\begin{array}{c}\text { Uso de } \\
\text { dispositivos } \\
\text { ahorradores }\end{array}$} & $\begin{array}{l}\text { Inodoros que presente sistema de } \\
\text { ahorro de agua. }\end{array}$ & $A-B-C$ & 72,00 a 352,22 & $3,49 \%$ a $96,66 \%$ \\
\hline & & $\begin{array}{l}\text { Grifería de lavamanos con sistema } \\
\text { de ahorro de agua. }\end{array}$ & $A-B-C$ & 30,55 a 375,37 & $2,86 \%$ a $87,59 \%$ \\
\hline & & $\begin{array}{c}\text { Grifería de fregadero con sistema } \\
\text { de ahorro de agua. }\end{array}$ & $A-B-C$ & 46,33 a 408,78 & $2,63 \%$ a $91,81 \%$ \\
\hline & & $\begin{array}{l}\text { Grifería de ducha con sistema de } \\
\text { ahorro de agua. }\end{array}$ & $A-B-C$ & 56,12 a 622,66 & $2,06 \%$ a $92,32 \%$ \\
\hline & $\begin{array}{c}\text { Uso de } \\
\text { vegetación } \\
\text { xerófita y/o } \\
\text { nativa }\end{array}$ & $\begin{array}{c}\text { Uso de vegetación apropiada en } \\
\text { jardines. }\end{array}$ & $A-B-C$ & 10,77 & $89,72 \%$ \\
\hline & \multirow{2}{*}{$\begin{array}{l}\text { Ahorro de } \\
\text { agua para } \\
\text { el riego de } \\
\text { jardines }\end{array}$} & $\begin{array}{c}\text { Sistema de control de riego por } \\
\text { goteo }\end{array}$ & $A-B-C$ & 136,75 & $17,11 \%$ \\
\hline & & $\begin{array}{l}\text { Red de distribución de riego por } \\
\text { goteo }\end{array}$ & $A-B-C$ & 2,58 & $92,85 \%$ \\
\hline & \multirow{2}{*}{$\begin{array}{l}\text { Sistema de } \\
\text { reutilización } \\
\text { de agua } \\
\text { Iluvia }\end{array}$} & $\begin{array}{l}\text { Sistema de presión para la } \\
\text { reutilización agua lluvias }\end{array}$ & $A-B-C$ & 1866,37 & $21,30 \%$ \\
\hline & & $\begin{array}{c}\text { Sistema de captación y } \\
\text { distribución de agua lluvia para el } \\
\text { uso en la vivienda. }\end{array}$ & $A-B-C$ & 992,09 a 1734,30 & $90,88 \%$ a $94,57 \%$ \\
\hline
\end{tabular}

Tabla 3. Resumen de las estrategias sustentables aplicadas en la etapa de construcción. Fuente: Elaboración de los autores. 


\section{RESULTADOS Y DISCUSIÓN}

\section{RESULTADOS DE LA ENCUESTA APLICADA (ANÁLISIS CUALITATIVO)}

Como resultado de la encuesta aplicada (análisis cualitativo) en las 280 viviendas seleccionadas del cantón Cuenca, se determinó lo siguiente:

- El $29 \%$ y el $32 \%$ de los encuestados consideran importante y muy importante respectivamente, que una vivienda presente características sustentables (Figura 3).

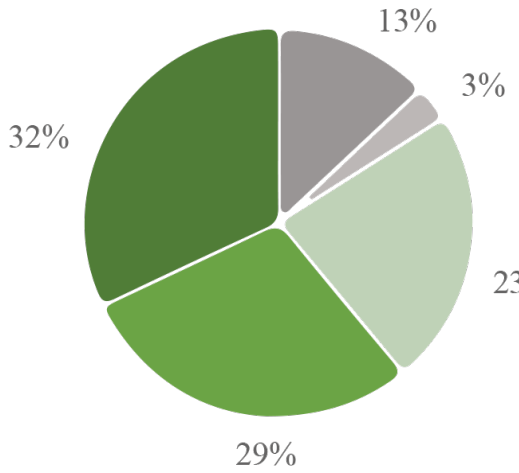

$$
\begin{aligned}
& \text { - Nada } \\
& \text { importante } \\
& \text { - Poco } \\
& \text { importante } \\
& \text { - Indiferente } \\
& \text { - Importante } \\
& \text { - Muy } \\
& \text { importante }
\end{aligned}
$$

Figura 3. Porcentaje de importancia de una vivienda sustentable en el cantón Cuenca. Fuente: Elaboración de autores.

- El $34 \%$ de la población total encuestada está dispuesta a invertir en una vivienda con características sustentables, cubriendo en promedio un $32 \%$ del valor adicional. La Figura 4 presenta los porcentajes de inversión según el nivel socio económico.
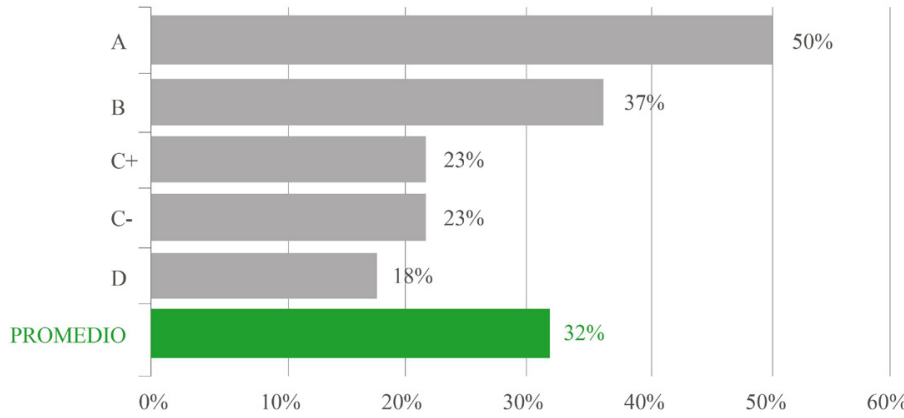

Figura 4. Porcentaje de inversión adicional a pagar por viviendas sustentables en Cuenca (clasificado por nivel socioeconómico). Fuente: Elaboración de autores.

\begin{tabular}{|c|c|c|c|}
\hline & Clas. & Desc. & Estrategias \\
\hline \multirow{17}{*}{ 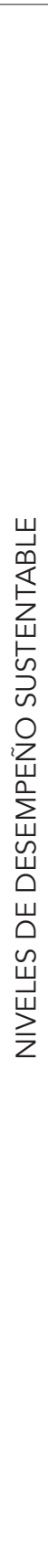 } & \multirow{6}{*}{ A } & \multirow{6}{*}{$\begin{array}{l}\text { Vivienda } \\
\text { Sustentable } \\
\text { Superior }\end{array}$} & $\begin{array}{l}\text { - Aplicación de tecnología a la } \\
\text { vivienda (domótica) }\end{array}$ \\
\hline & & & $\begin{array}{c}\text { - Implementación de fuentes de } \\
\text { energía renovables }\end{array}$ \\
\hline & & & $\begin{array}{l}\text { - Aplicación de diseño } \\
\text { bioclimático a la envolvente }\end{array}$ \\
\hline & & & $\begin{array}{c}\text { - Uso de dispositivos ahorradores } \\
\text { de agua (práctica superior) }\end{array}$ \\
\hline & & & $\begin{array}{l}\text { - Uso de vegetación xerófita y/o } \\
\text { nativa (práctica superior) }\end{array}$ \\
\hline & & & $\begin{array}{l}\text { - Sistema de reutilización de } \\
\text { agua lluvia (práctica superior) }\end{array}$ \\
\hline & \multirow{6}{*}{ B } & \multirow{6}{*}{$\begin{array}{l}\text { Vivienda } \\
\text { Sustentable } \\
\text { Mejor }\end{array}$} & $\begin{array}{l}\text { - Uso de luminaria focos } \\
\text { ahorradores, fluorescentes o led }\end{array}$ \\
\hline & & & $\begin{array}{l}\text { - Aplicación de diseño } \\
\text { bioclimático a la envolvente }\end{array}$ \\
\hline & & & $\begin{array}{l}\text { - Uso de dispositivos ahorradores } \\
\text { de agua (nivel mejores prácticas) }\end{array}$ \\
\hline & & & $\begin{array}{l}\text { - Uso de vegetación xerófita y/o } \\
\text { nativa en el predio (nivel mejores } \\
\text { prácticas) }\end{array}$ \\
\hline & & & $\begin{array}{l}\text { - Ahorro de agua para riego de } \\
\text { jardines (mejores prácticas) }\end{array}$ \\
\hline & & & $\begin{array}{l}\text { - Sistema de reutilización de } \\
\text { agua lluvia (mejores prácticas) }\end{array}$ \\
\hline & \multirow{5}{*}{ C } & \multirow{5}{*}{$\begin{array}{l}\text { Vivienda } \\
\text { Sustentable } \\
\text { Estándar }\end{array}$} & $\begin{array}{l}\text { - Uso de luminaria focos } \\
\text { ahorradores, fluorescentes o led }\end{array}$ \\
\hline & & & $\begin{array}{c}\text { - Uso de dispositivos ahorradores } \\
\text { de agua (práctica estándar) }\end{array}$ \\
\hline & & & $\begin{array}{l}\text { - Uso de vegetación xerófita } \\
\text { y/o nativa en el predio (práctica } \\
\text { estándar) }\end{array}$ \\
\hline & & & $\begin{array}{l}\text { - Ahorro de agua para riego de } \\
\text { jardines (práctica estándar) }\end{array}$ \\
\hline & & & $\begin{array}{l}\text { - Sistema de reutilización de } \\
\text { agua lluvia (práctica estándar) }\end{array}$ \\
\hline & D & $\begin{array}{l}\text { Vivienda } \\
\text { Convencional }\end{array}$ & \\
\hline
\end{tabular}

\section{RESULTADOS DEL CASO DE ESTUDIO (ANÁLISIS CUANTITATIVO).}

Para determinar los niveles de desempeño de la vivienda sustentable, se analizaron las estrategias establecidas por Baquero y Quesada (2016), Calle y Ortiz (2016) y Molina y Orellana (2017) con las cuales se obtiene una clasificación que se presenta en la Tabla 4.

Tabla 4. Clasificación de las viviendas sustentables respecto a la aplicación de estrategias obtenidas para Cuenca. Fuente: Elaboración de los autores. 


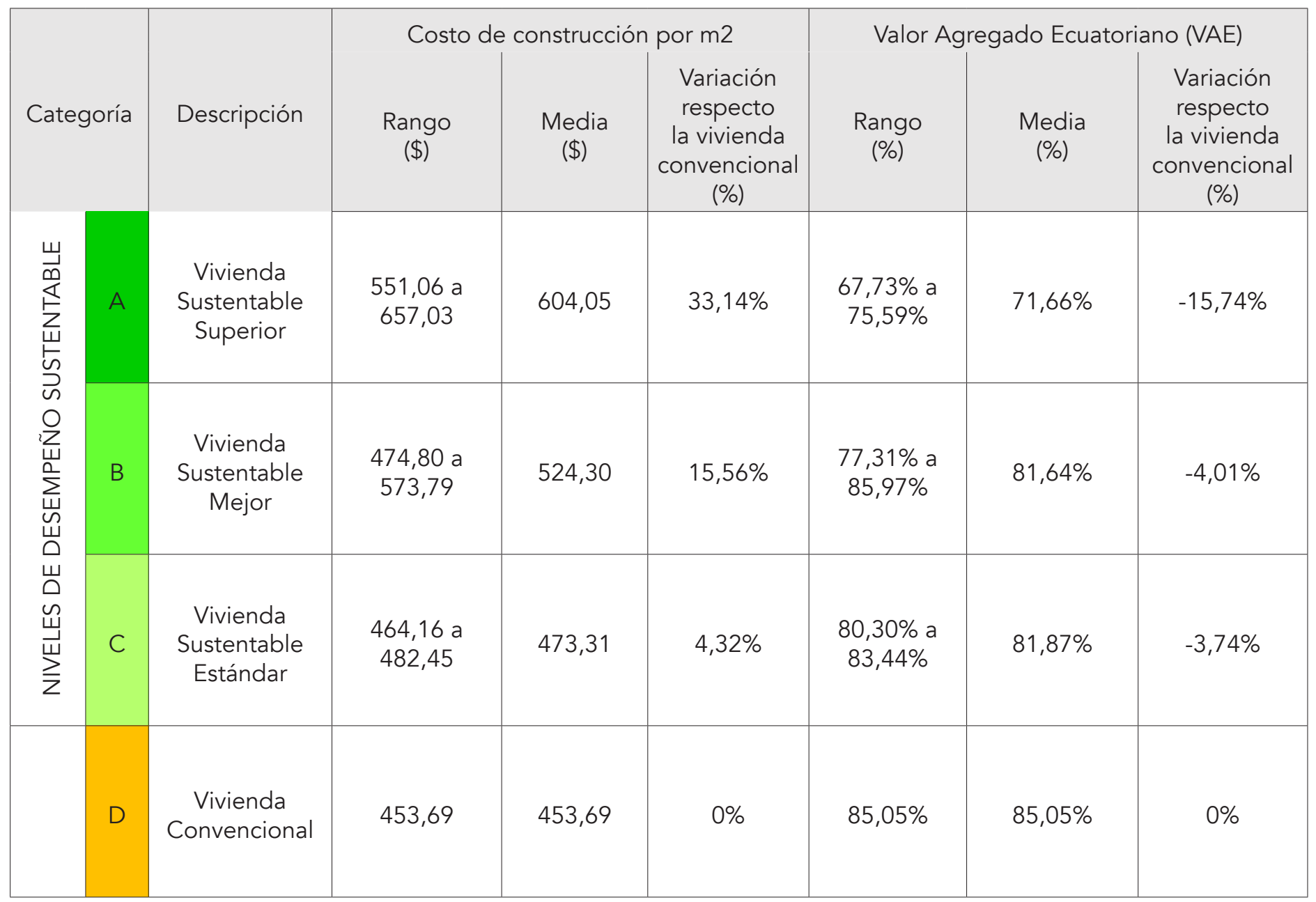

Tabla 5. Costo de construcción, VAE determinado respecto a la clasificación de las viviendas sustentables en Cuenca-Ecuador. Fuente: Elaboración de autores.

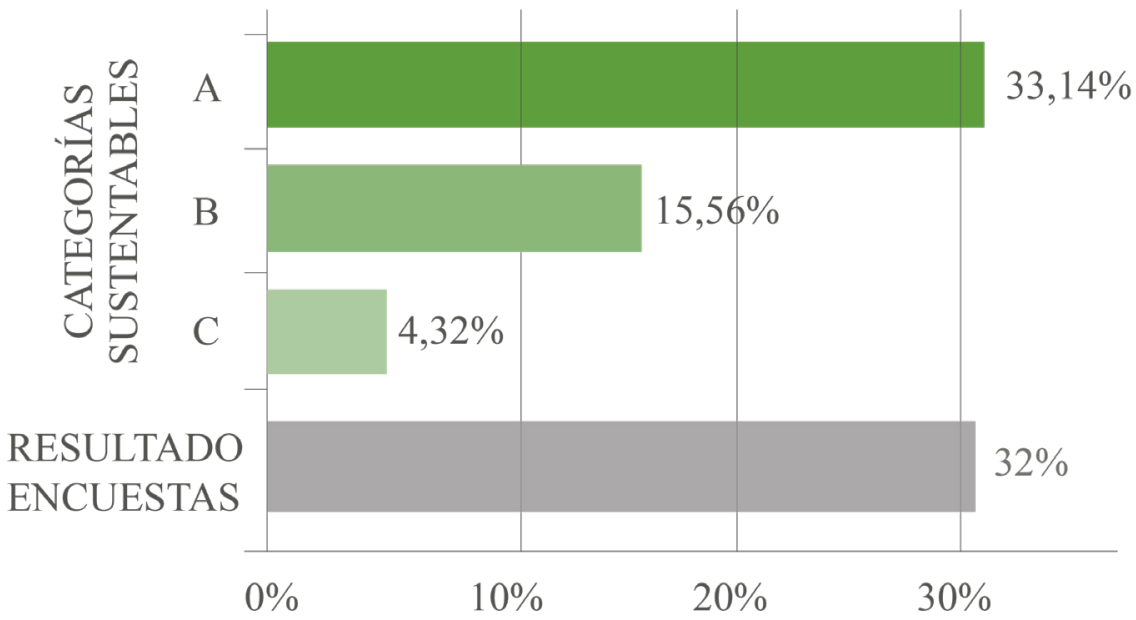

Figura 5. Comparación porcentual entre los resultados del análisis cualitativo y el análisis cuantitativo.

Fuente: Elaboración de autores. 


\section{Costos de la vivienda convencional.}

El costo de construcción (por metro cuadrado) y el VAE de la vivienda unifamiliar convencional (caso de estudio) se establecen en $\$ 453,69$ USD (dólares americanos) y en el $85,05 \%$, respectivamente. Estos valores son considerados como base de comparación para fijar las variaciones de los valores semejantes de las viviendas sustentables en las diferentes categorías.

\section{Variación del costo de construcción y del VAE según los niveles de desempeño sustentable.}

La Tabla 5 detalla, según la clasificación de la vivienda unifamiliar del Cantón Cuenca, los costos de construcción (por metro cuadrado) y el VAE. Los resultados obtenidos se presentan por medio de su rango o límites, el valor promedio y la variación respecto al valor base semejante de la vivienda unifamiliar convencional.

A partir de los resultados obtenidos, se establece que la vivienda unifamiliar sustentable requiere inversiones adicionales en su construcción entre el $4.32 \%$ en su clasificación estándar al 33.14\% en su clasificación Superior. El VAE en las viviendas sustentables, presenta valores semejantes $81.87 \%$ y $81.64 \%$ en las categorías Estándar y Mejor, respectivamente; valor que desciende al $71.66 \%$ en la categoría Superior.

\section{ANÁLISIS DE RESULTADOS}

En Cuenca, las personas interesadas en invertir en una vivienda unifamiliar sustentable están dispuestas a cubrir un costo adicional del $32 \%$, en promedio, sobre el costo de su semejante convencional. Los costos de construcción de este tipo de viviendas son más altos que su semejante convencional, pues requieren una inversión adicional del 33.14\%, 15.56\% y $4.32 \%$ correspondientes a las categorías Superior (A), Mejor (B) y Estándar (C). Si bien la predisposición de la población no cubre el incremento promedio de las viviendas referenciales sustentables en la categoría Superior (A), sí son aceptadas por sus potenciales compradores las categorías Mejor (B) y Estándar (C), según se puede observar en la Figura 5 .

Según los resultados presentes en la Tabla 5, todos los valores calculados del VAE en las diferentes categorías de viviendas unifamiliares sustentables son menores al estimado para la vivienda unifamiliar convencional, debido a que, para aprovechar los recursos ambientales presentes en la localidad, reducir el consumo de energía y brindar un mejor entorno de vivienda, es imprescindible el empleo de materiales y equipos especializados, de origen extranjero.

\section{CONCLUSIONES}

Este estudio permitió determinar que en Cuenca un alto porcentaje de su población está dispuesto a invertir en una vivienda con características sustentables, a pesar de que los costos de construcción de las viviendas unifamiliares sustentables sí representan un incremento con respecto a sus semejantes convencionales. No obstante, al requerir una inversión adicional mínima del $4,32 \%$, se considera factible su construcción, ya que el costo de incremento es menor al de la predisposición de inversión local.

Se pudo establecer, asimismo, una estimación de la inversión inicial del costo por la aplicación de las estrategias sustentables, el que en una futura investigación podría ser comparado con los beneficios relacionados al ciclo de vida de la edificación para, en definitiva, precisar el costo de la vivienda sustentable.

Por último, se estableció que el VAE de todas las categorías de viviendas sustentables es menor a su semejante convencional, debido a que en gran parte los materiales y equipos deben ser de origen extranjero, pues no existe producción nacional.

\section{AGRADECIMIENTOS}

El estudio está basado en los resultados de la tesis para obtener el título de magíster en la Maestría en Construcciones del Centro de Postgrados de la Facultad de Arquitectura y Urbanismo de la Universidad de Cuenca. Agradecemos el apoyo del proyecto de investigación "Método de Certificación de la Construcción Sustentable de Viviendas", del cual forma parte la presente investigación.

\section{REFERENCIAS BIBLIOGRÁFICAS}

ALSHAMRANI, Othman Subhi. Construction cost prediction model for conventional and sustainable college buildings in North America. Journal of Taibah University for Science, 2017, $\mathrm{n}^{\circ}$ 11, pp. 315-323.

ASAMBLEA CONSTITUYENTE DEL ECUADOR. Constitución del Ecuador. In., 2008

ASSAD, Minass, HOSNY, Ossama, ELHAKEEM, Ahmed y EL HAGGAR, Salah. Green building design in Egypt from cost and energy perspectives. Architectural Engineering and Design Management, 2015, $\mathrm{n}^{\circ} 11$, pp. 21-40. 
BAQUERO, María Teresa y QUESADA, Felipe. Eficiencia energética en el sector residencial de la ciudad de Cuenca, Ecuador. Maskana, 2016, n 7, pp. 147-165.

BARTLETT, Ed y HOWARD, Nigel. Informing the decision makers on the cost and value of green building. Building Research \& Information, 2000, $\mathrm{n}^{\circ} 28$, pp. 315-324.

BERARDI, Umberto. Clarifying the new interpretations of the concept of sustainable building. Sustainable Cities and Society, 2013, n 8 , pp. 72-78.

CALLE, Andrea y ORTIZ, Jessica. Evaluación del consumo energético en la fase de uso de la edificación residencial en Cuenca: Diseño de estrategias para un modelo de vivienda eficiente. Tesis de pregrado. Universidad de Cuenca, Cuenca, 2016.

CÁMARA DE LA CONSTRUCCIÓN DE CUENCA. Boletín técnico. Cuenca, Ecuador, 2016.

CONSEIL INTERNATIONAL DU BÂTIMENT (CIB). Towards sustainable and smart-ecobuildings. Rotterdam, the Netherlands, 2010 .

EXA, Agencia espacial civil ecuatoriana. Estación climática Cuenca [en línea]. [Consultado 02 diciembre 2017]. Disponible en: http://cue.exa.ec/

FULLBROOK, D.; JACKSON, Q. y FINLAY, G. Value Case for sustainable building in New Zealand. Wellington, New Zealand: 2006 .

GARCÍA ERVITI, Federico; ARMENGOT PARADINAS, Jaime y RAMÍREZ PACHECO, G. El análisis del coste del ciclo de vida como herramienta para la evaluación económica de la edificación sostenible. Estado de la cuestión. Informes de la Construcción, 2015, n 67, pp. 1-8.

GUNAWANSA, Asanga y KUA, Harn Wei. A comparison of climate change mitigation and adptation strategies for the construction industries of three coastal territories. Sustainable development, $2014, n^{\circ}, p p$.

HWANG, Bon-Gang y TAN, Jac See. Sustainable Project Management for Green Construction: Challenges, Impact and Solutions. En: World Construction conference - Global challenges in construction industry. Colombo: World Construction conference, June 2012, pp. 171-179.

INSTITUTO NACIONAL DE EFICIENCIA ENERGÉTICA Y ENERGÍAS RENOVABLES (INER). Edificaciones presentación. Quito: 2014.

INSTITUTO NACIONAL DE ESTADÍSTICAS Y CENSOS (INEC). Resultados del Censo 2010 de población y vivienda en el Ecuador. Quito: INEC, 2010a.

INSTITUTO NACIONAL DE ESTADÍSTICAS Y CENSOS (INEC). Fascículo provincial del azuay. Ecuador: 2010b.

KARATAS, Aslihan y EL-RAYES, Khaled. Optimizing tradeoffs among housing sustainability objectives. Automation in Construction, 2015, n 53 , pp. 83-94.
KATS, Gregory; ALEVANTIS, Leon; BERMAN, Adam; MILLS, Evan y PERLMAN, Jeff. The cost and financial benefits of green buildings: A report to California's sustainable building task force. California: 2003

KATS, Gregory; BRAMAN, Jon y JAMES, Michael Greening our built world: costs, benefits, and strategies. Washington, DC: Island Press, 2010.

KNEIFEL, Joshua. Life-cycle carbon and cost analysis of energy efficiency measures in new commercial buildings. Energy and Buildings, 2010, $\mathrm{n}^{\circ} 42$, pp. 333-340.

MOLINA, Eduardo y ORELLANA, Diana. Sustentabilidad de agua en viviendas de la ciudad de Cuenca. Tesis de magíster inédita Universidad de Cuenca, Cuenca, 2017

ONEILL, Kirstie J. y GIBBS, David C. Towards a sustainable economy? Socio-technical transitions in the green building sector. Local Environment, 2014, n²19, pp. 572-590.

QUESADA, F. Métodos de evaluación sostenible de la vivienda: Análisis comparativo de cinco métodos internacionales. Revista Hábitat Sustentable, 2014, nº 4, pp. 56-67.

REHM, Michael y ADE, Rochelle. Construction costs comparison between 'green' and conventional office buildings. Building Research \& Information, 2013, n 41, pp. 198-208.

SECRETARÍA NACIONAL DE CONTRATACIÓN PÚBLICA (SERCOP). Metodología para determinar el valor agregado ecuatoriano por produto en los procedimientos de contratación pública de bienes. Quito, 2015a. RESOLUCIÓN N. RESERCOP-2015-0000031.

SECRETARÍA NACIONAL DE CONTRATACIÓN PÚBLICA (SERCOP). Metodología para la definición de una oferta como ecuatoriana en los procesos de adquisición de bienes y prestación de servicios. Quito, 2015b. Resolución Nº R.E. SERCOP-2015-000033.

UMAR, Usman; KHAMIDI, Mohd y TUKUR, Hassan 2012. Sustainable building material for green building construction, conservation and refurbishing. En: U.T.D.M. UTM ed. Management in construction research association: Postgraduate conference. Malasia: UTM2012, pp.

UNEP SBCl, Sustainable buildings and climate initiative. Buildings and climate change: Summary for decision-makers. París, Francia: U.N.E. PROGRAMME, 2009.

URBAN GREEN COUNCIL. Cost of green in NYC [en línea]. [Consultado 02 diciembre 2017]. Disponible en: http://blog.urbangreencouncil.org/wp-content/ uploads/2012/03/Cost_Study_Full_Download. pdf?_ga $=2.161901052 .1300835507 .1513780553$. 1076632804.1513780553

URIBE, Eduardo. El cambio climático y sus efectos en la biodiversidad en América Latina. Naciones Unidas, Santiago: CEPAL, 2015.

VIVIENDA SUSTENTABLE Y SEGURA CUENCA. Metodología de la evaluación [en línea]. [Consultado 08 marzo 2017]. Disponible en: http://viviendasustentablecuenca.com/certificacion/ metodologia/ 
WILCOXON, Frank. Individual Comparisons by Ranking Methods. Biometrics Bulletin, 1945, n 1, pp. 80-83.

ZORE, Žan, ČUČEK, Lidija y KRAVANJA, Zdravko. Syntheses of sustainable supply networks with a new composite criterion Sustainability profit. Computers \& Chemical Engineering, 2017, $n^{\circ} 102$, pp. 139-155. 